\title{
Constructive and Destructive Interference of Acoustic and Entropy Waves in a Premixed Combustor with a Choked Exit
}

\author{
Wolfgang Polifke \\ Lehrstuhl für Thermodynamik, Technische Universität München, D-85747 Garching, Germany \\ Christian Oliver Paschereit and Klaus Döbbeling \\ Alstom Power Ltd., CH-5405 Baden, Switzerland
}

(Received 8 September 1999; revised 27 September 2000; accepted 25 January 2001)

\begin{abstract}
Thermoacoustic instabilities are a cause for concern in combustion applications as diverse as small household burners, gas turbines and rocket engines. In this work, a feedback mechanism is analysed, which couples combustion chamber acoustics with convectively transported fluctuations of entropy (entropy waves) generated within a premixed flame. Essential elements of this thermo-acoustic feedback loop are: 1) fluctuations in fuel concentration, induced by acoustic disturbances at the location of fuel injection, 2) convective transport of fuel inhomogeneities through the premixing section of the burner, 3) modulations in heat release rate and hot gas entropy resulting from the consumption of fuel/air mixture with varying fuel concentration by the flame, and 4) the generation of sound through entropy non-uniformities at the turbine inlet. From a qualitative analysis based on relative phases, it is concluded that, depending on the various convective and acoustic time lags involved, entropy waves may couple constructively as well as destructively with combustor acoustics. However, such qualitative analysis does not indicate whether the coupling between entropy and acoustic waves is strong enough to significantly influence thermo-acoustic stability. Therefore, a linear model has been constructed to estimate the effect of entropy waves on the thermo-acoustic response and stability of a combustor with a choked exit nozzle, as it might be found in a gas turbine. Note that phenomena like dispersion of convective waves, distributed heat release, vortical velocities, etc., have not been taken into account, as they would burden the presentation with unnecessary complexity. Results obtained indicate that the interaction between combustor acoustics and entropy waves can be significant, especially for the lowest non-axisymmetric modes, and even at frequencies higher than those usually associated with convective waves. As expected, it was observed that the coupling between pressure and entropy waves at the exit nozzle can enhance as well as reduce the thermo-acoustic stability of a combustor, or the responsiveness to an external or internal fluid-mechanic excitation mechanism. It is concluded that a comprehensive thermo-acoustic analysis of a premixed combustor with a choked exit must in general include the generation and propagation of entropy waves and the coupling with combustor acoustics.
\end{abstract}

\section{INTRODUCTION}

It has been realised quite some time ago that the flow of gas containing regions of non-uniform temperature ("entropy spots") through a nozzle can be a significant source of combustion noise. ${ }^{1}$ A comprehensive review of earlier work on this subject has been compiled by Marble and Candel. ${ }^{2}$

It has also been understood that convection of entropy spots into a nozzle or more generally a choked cross section can result in self-excited combustion instability. ${ }^{3-9}$ Much of the earlier work concentrated on ramjet combustors. See the review by Culick. ${ }^{5}$ More recently, attention has been turned towards (premixed) gas turbine combustors; see the work by Keller et al. ${ }^{4,7}$ and Dowling and co-workers. ${ }^{6,8}$ Because entropy spots are transported by the mean flow, the frequencies associated with these instabilities have been argued to be generally "low", i.e. lower than the acoustic eigenfrequencies of the combustor.

In the present work it is emphasised that in compact premixed combustors, as they are commonly encountered in low-emission combustion systems, acoustic oscillations and entropy fluctuations couple with each other even at "high" frequencies, i.e. frequencies corresponding to acoustic eigenmodes. This coupling is achieved through a multiply-connected feedback mechanism involving fluctuations of heatrelease-rate - pressure - velocity - fuel concentration and entropy. Fluctuations of entropy are generated as fuel inhomogeneities that are consumed by the flame. Note that this scenario is quite distinct from, e.g., the analysis of entropy fluctuations in a ramjet combustor by Humphrey and Culick, ${ }^{10}$ where entropy fluctuations upstream of the flame drive the instability mechanism. The amplitude of these entropy waves is either imposed as an upstream boundary condition, or produced by pressure waves incident on the inlet shock.

Analysing the various convective and acoustic time lags involved in the aforementioned feedback mechanism, we arrive at a conclusion, which to our knowledge has not been emphasised previously: entropy waves may couple constructively as well as destructively with combustor acoustics. In other words, the interaction of pressure and entropy waves can enhance as well as reduce the thermo-acoustic stability of a combustor or the responsiveness to an external or internal fluid-mechanic excitation mechanism. 